\title{
Numerical Simulation of Multiphase Flow Structures in Openfoam Software Package
}

\author{
E. A. Ivanov ${ }^{1^{*}}, A . S$. Klyuyev ${ }^{1}, A . A$. Zharkovskii $^{1}$ and $I . O$. Borshchev ${ }^{1}$ \\ ${ }^{1}$ Peter the Great St.Petersburg Polytechnic University, St.Petersburg, Russia
}

\begin{abstract}
Numerical simulation of various structures of multiphase flow in the pipe was performed using the OpenFOAM software package. A visual comparison of multiphase flow design structures for separated stratifiedwave, plug and annular flow modes with experimental data is presented. For multiphase flow modelling the solver compressibleInterFoam was used. From the results of numerical modelling, it follows that the OpenFOAM software package allows correct prediction of multiphase flow modes in the pipe depending on Reynolds numbers for gas and liquid phases of the flow.
\end{abstract}

\section{Introduction}

Mathematical modeling of multiphase flows in branched pipeline networks is complicated by the variety of flow modes, depending on the velocities of liquid and gas phases, as well as on pipeline position in space (horizontal configuration, angular configuration, vertical configuration of pipeline section). Besides, simulation is complicated by possible local network objects, such as gate valves, valves, etc. Therefore, existing empirical approaches to simulation are very limited by the scope of application $[1,2,4,5]$.

At present, 3D computation methods based on finite volume method (hereinafter referred to as FVM) are widely used for hydrodynamics modeling $[5,6]$. When using FVM, the mathematical model is more universal than empirical or one-dimensional computation methods. FVM mathematical model, verified on simple tasks, can be applied to tasks with more complex geometric shape.

For numeric computation of multiphase flow in branched pipeline network, freely propagating OpenFOAM library, delivered with open-source code in $\mathrm{C}++$ programming language, was selected. This library allows to perform numerical simulations using the finite volume method and has built-in solvers for simulation of fluid and gas dynamics of multiphase flows.

* Corresponding author: ivanov_ea@spbstu.ru 


\section{Description of multiphase flow structures in pipeline sections}

In scientific papers $[1,2]$, schemes of possible structures of multiphase flow in horizontal pipeline sections are presented. The scientific paper by Afshin J. Ghajar and Clement C. Tang [2] describes 8 different flow structures depending on Reynolds number for each phase. These structures of multiphase flow in horizontal pipeline are shown in figure 1.

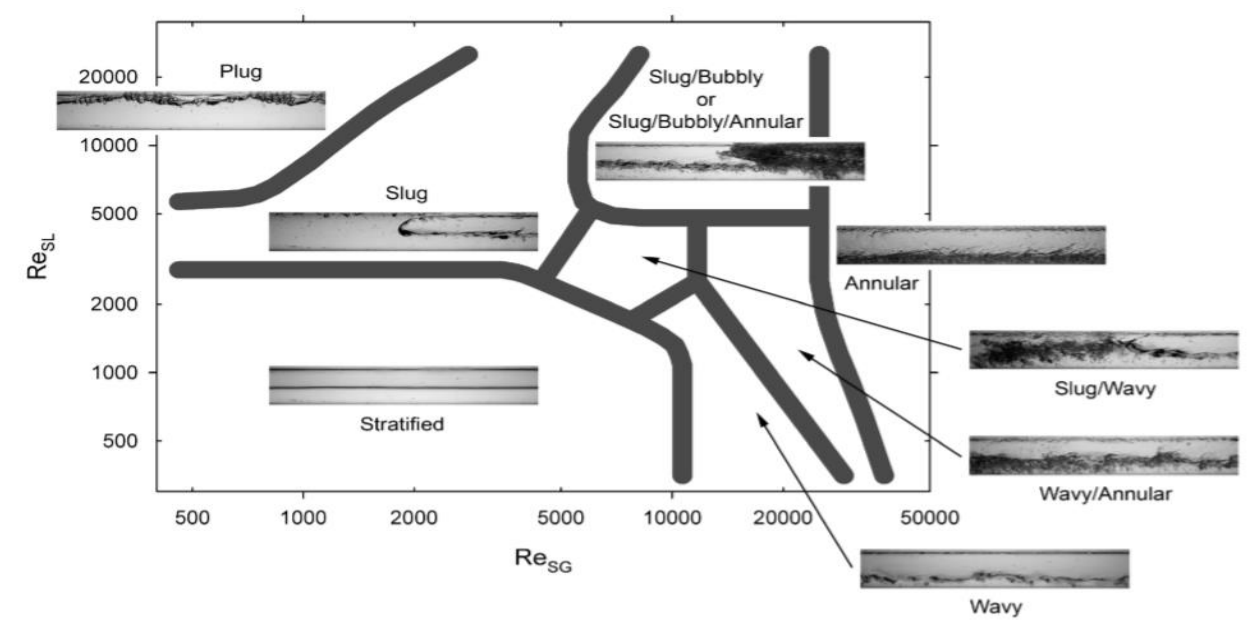

Fig. 1. The structure of multiphase flow in horizontal pipeline in the scientific paper by Afshin J. Ghajar and Clement C. Tang [2].

In research literature "Hydraulics of two-phase flows in pipelines", Maron V. I. [1], the author combines intermediate flow structures and leaves only 4 fundamentally different flow structures, which are shown in figure 2.

Plumb structure

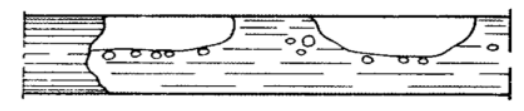

Stratified structure
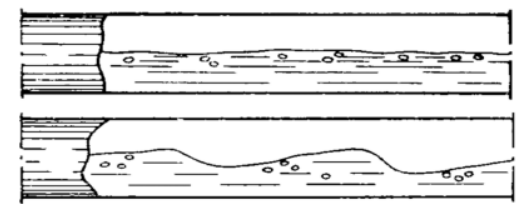

Annular structure

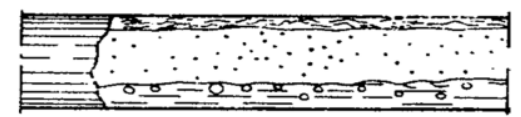

Gas

Slug structure

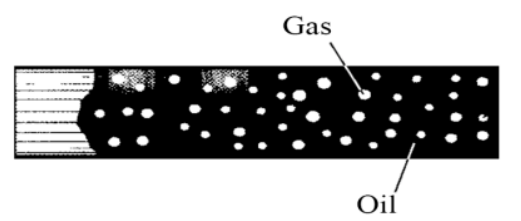

Fig. 2. Multiphase flow structures by Maron V. I. 


\section{Mathematical model description}

CompressibleInterFoam solver, a part of the OpenFOAM libraries, was used to simulate the multiphase flow.

CompressibleInterFoam solver is used to simulate heterogeneous compressible twophase flows. This solver uses VOF (Volume of fluid) method to track the interface between liquid and gas phases of the flow. Viscosity, density, and specific heat capacity of the flow are determined for gas liquid mixture. Where gas-liquid mixture volume concentration $\alpha$ can have a value from 0 to 1 , where at $\alpha=1$ the gas-liquid mixture assumes liquid properties, and at $\alpha=0$ gas properties. The solver includes the following equations for gas-liquid mixture: motion equation, equation of continuity, energy equation, and transfer equation for volume concentration value $\alpha$. The listed equations of compressibleInterFoam solver are presented below:

The equation of continuity:

$$
\frac{\partial \rho}{\partial t}+\nabla(\rho U)=0
$$

The motion equation:

$$
\frac{\partial \rho U}{\partial t}+\nabla(\rho U U)=-\nabla p+\nabla(\mu \nabla U)+S_{U}
$$

The energy equation:

$$
\frac{\partial\left(\rho C_{p} T\right)}{\partial t}+\nabla\left(\rho U C_{p} T\right)=\nabla(k \nabla T)+S_{T}
$$

The transport equation of volume concentration:

$$
\frac{\partial \alpha}{\partial t}=\nabla(\alpha U)+\nabla\left[(1-\alpha) \alpha U_{r}\right]=0,
$$

where $\rho$-density, $U$ - velocity, $t$ - time, $p$ - pressure, $\mu$-dynamic viscosity, $C_{p}$ - specific heat,

$T$-temperature, $k$-thermal conductivity, $S_{U}$ and $S_{T}$-additional sources in the motion equation and in the energy equation.

To solve the equations described above, the finite volume method with the PIMPLE solution algorithm [3] is used.

\section{Problem set up description}

Design geometry is a pipeline section with diameter $\mathrm{D}=25.4 \mathrm{~mm}$ and a relative pipe length of $\mathrm{L} / \mathrm{D}=214$. Design geometry visualization is shown in figure 3 .

Fig. 3. Design geometry of the pipeline section. 
Analysis grid is constructed in Salome non-commercial software package, the analysis grid is structured, elements type - hexahedrons. To save computing time, the grid is built rough -90 thousand elements of the analysis grid. Then, during computation process, the adaptive grid condensation method was applied based on tracking the interface boundary between liquid and gas phases. Adaptive analysis grid visualization is shown in figure 4 . The upper limit of the elements number in the adaptive condensation of the grid comprised 1 million elements.

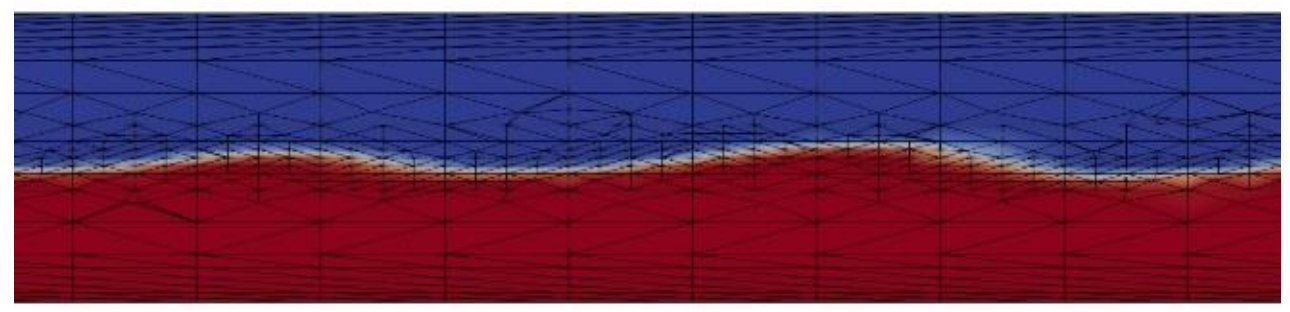

Fig. 4. Adaptive analysis grid visualization during computation

As the boundary conditions at the pipe inlet, the $U$ velocity was set for each phase, corresponding to a certain Reynolds number. The walls were considered stationary, and the static pressure at the outlet was set to $p=1 \mathrm{~atm}$. Since gas compressibility was considered in the computation, the inlet temperature was set to $20^{\circ} \mathrm{C}$.

The formation of plug flow structure occurs mainly due to the existing disturbances in the flow associated with various external factors creating fluctuation of flow parameters, such as pumps, valves, complex pipeline geometry, etc. Therefore, for the formation of plug flow structure in the computation, a certain flow disturbance at the inlet is a necessary condition. In this regard, periodic supply of the gas or liquid phase with $10 \mathrm{~Hz}$ frequency is set at the inlet.

Incompressible water with $998 \mathrm{~kg} / \mathrm{m}^{3}$ density and $1 \mathrm{e}-06 \mathrm{~m}^{2} / \mathrm{s}$ kinematic viscosity was used as the liquid phase in the computation.

Nonviscous air under normal conditions of $1 \mathrm{~atm}, 20^{\circ} \mathrm{C}$ was used as gas.

\section{Numerical simulation results}

Table 1 shows the flow modes, computed in OpenFOAM environment.

Table 1. Flow regimes.

\begin{tabular}{|c|c|c|}
\hline Flow regime & $\begin{array}{c}\text { Liquid phase Reynolds number, } \\
\mathbf{R e}_{\mathbf{l}}\end{array}$ & $\begin{array}{c}\text { Gas phase Reynolds } \\
\text { number, } \mathbf{R e}_{\mathbf{g}}\end{array}$ \\
\hline Stratified structure & 705 & 832 \\
\hline Stratified-wave structure & 1500 & 16000 \\
\hline Plug structure & 17000 & 4000 \\
\hline Annular structure & 17000 & 28000 \\
\hline
\end{tabular}


Computation-experiment visual comparison was performed for the presented flow regimes. The results of the experimental data were borrowed from a scientific paper by Afshin J., Ghajar and Clement C. Tang, [2]. flow.

Figures 5 and 6 provide a visual comparison of the separate flow structure of two-phase

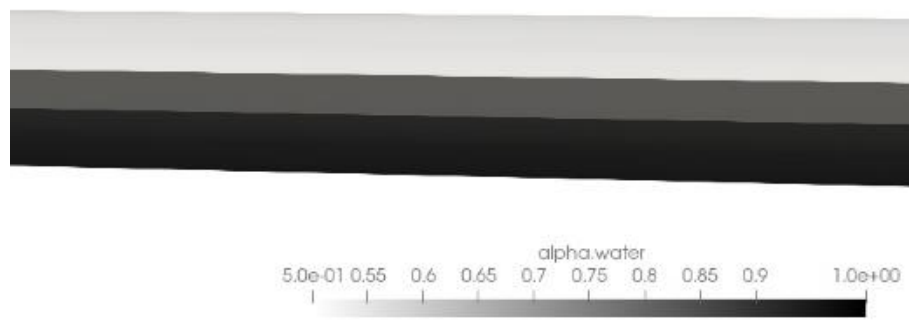

Fig. 5. Stratified flow structure, computation; $\operatorname{Re}_{\mathrm{g}}=832, \mathrm{Re}_{\mathrm{l}}=705$.

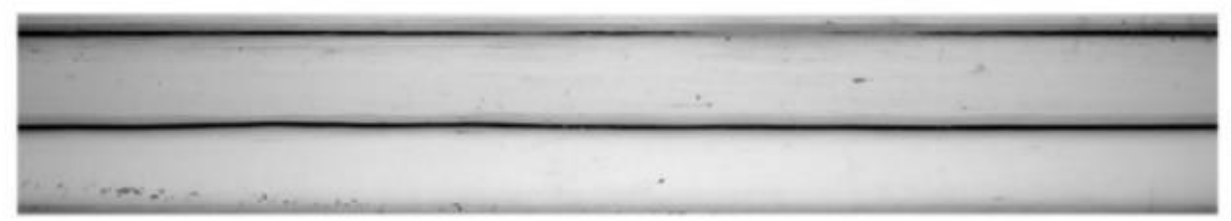

Fig. 6. Stratified flow structure, experiment; $\mathrm{Re}_{\mathrm{g}}=832, \mathrm{Re}_{\mathrm{l}}=705$.

Figures 5 and 6 demonstrate that the flow structure in both cases has clear phase boundary in the center of the pipe. At these Reynolds numbers, the flow for both phases is laminar.

Figures 7 and 8 provide visual comparison of the stratified-wave structure of two-phase flow.

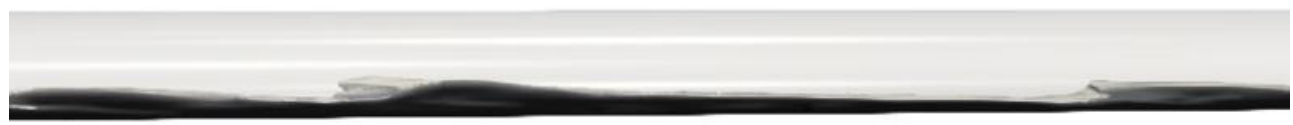

Fig. 7. Stratified-wave structure of the flow, computation; $\operatorname{Re}_{\mathrm{g}}=16000, \mathrm{Re}_{\mathrm{l}}=1500$.

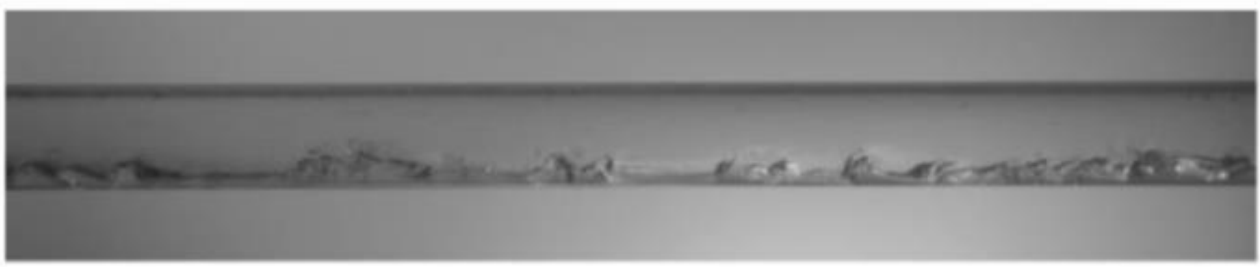

Fig. 8. Stratified-wave structure of the flow, experiment; $\mathrm{Re}_{\mathrm{g}}=16000, \mathrm{Re}_{\mathrm{l}}=1500$.

Figures 7 and 8 prove that gas phase turbulent flow forms periodic waves at the interface between liquid and gas phases. The computation demonstrates smoother wave structure, it is assumed that this is due to insufficient density of the analysis grid.

Figures 5 and 6 provide visual comparison of plug flow structure of two-phase flow. 


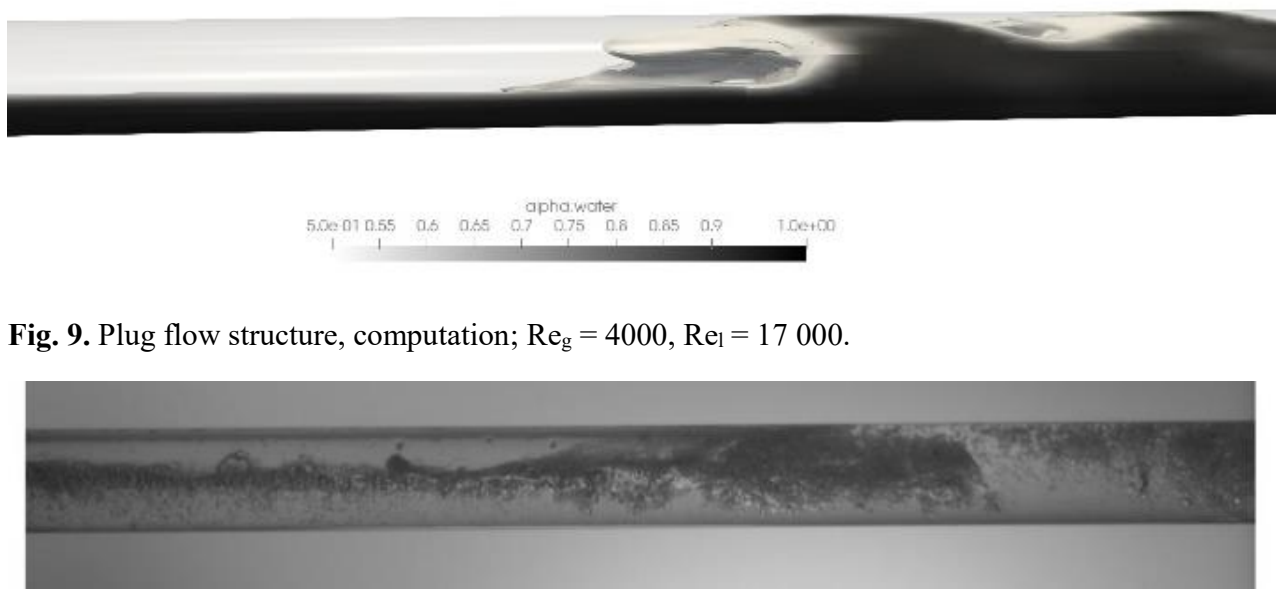

Fig. 10. Plug flow structure, experiment; $R e_{g}=4000, R_{1}=17000$.

Figures 9 and 10 demonstrate that liquid phase turbulent flow forms plug flow structures in the flow both in the computational model and in the experiment. However, the insufficient resolution of the analysis grid does not allow for detailed resolution of individual air bubbles present in the experiment.

Figures 11 and 12 provide visual comparison of the $\mathrm{r}$ annular structure of two-phase flow.
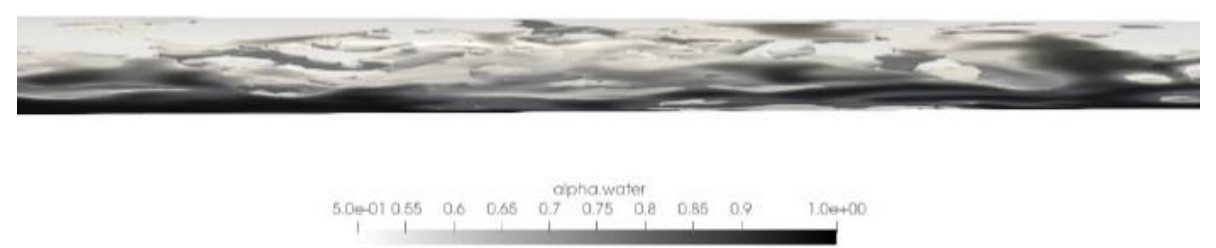

Fig. 11. The annular structure of the flow, computation; $\operatorname{Re}_{\mathrm{g}}=28000, \mathrm{Re}_{\mathrm{l}}=17000$.

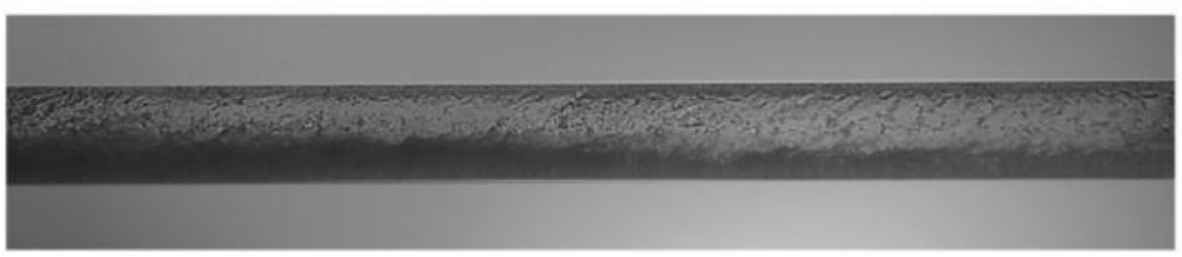

Fig. 12. The annular structure of the flow, experiment; $\operatorname{Re}_{\mathrm{g}}=28000, \mathrm{Re}_{\mathrm{l}}=17000$.

Figures 11, 12 prove that the Reynolds numbers $\mathrm{Re}_{\mathrm{g}}=28000$ and $\mathrm{Re}_{\mathrm{l}}=17000$ correspond to the annular structure of the flow for both calculation and experiment.

\section{Conclusions}

Conducted numerical studies prove that compressibleInterFoam solver of OpenFOAM software package allows to simulate various structures of multiphase flow: stratified, stratified-wave, plug, annular. Numerical simulation results are confirmed by visual comparison with the experimental data. However, for more accurate simulation of flow details, significant grid clustering is required, which in turn requires significant 
computational resources. It is worth noting that the quantitative assessment of the multiphase flow is complicated by strongly expressed non-stationary nature of this process.

\section{References}

1. V. I. Maron, Hydraulics of Two-Phase Flows in Pipelines (Saint Petersburg: Lan'), 256 (2012)

2. A. J. Ghajar and Tang C. C. Heat transfer measurements, flow pattern maps, and flow visualization for non-boiling two-phase flow in horizontal and slightly inclined pipe Heat Transfer Engineering vol 28 ( Taylor \& Francis Group ) pp 525-40, 2007

3. C. J. Greenshields, OpenFOAM User Guide version 8 OpenFOAM Foundation Ltd, 243 (2020)

4. Y. Taitel and Dukler A. E. A model for predicting flow regime transitions in horizontal and near horizontal gas-liquid flow AIChE Journal 22 47-55, 1976

5. J. M. Mandhane, Gregory G. A. and Aziz K.. A flow pattern map for gas-liquid flow in horizontal pipes International Journal of Multiphase Flow 1 537-53, 1974

6. V. E. Shcherba, Shalai V. V. and Zanin A. V. Thermodynamic Foundations of Working Processes in Positive-Displacement Machines Designed for Compression of Two-Phase, Two-Component Working Fluids Chemical and Petroleum Engineering 55 463-72, 2019 